\title{
CFTR (ABCC7) is a hydrolyzable-ligand-gated channel
}

\author{
Andrei A. Aleksandrov • Luba A. Aleksandrov • \\ John R. Riordan
}

Received: 22 June 2006 / Revised: 13 July 2006 / Accepted: 19 July 2006 / Published online: 26 September 2006

(C) Springer-Verlag 2006

\begin{abstract}
As the product of the gene mutated in cystic fibrosis, the most common genetic disease of Caucasians, CFTR is an atypical ABC protein. From an evolutionary perspective, it is apparently a relatively young member of the $\mathrm{ABC}$ family, present only in metazoans where it plays a critical role in epithelial salt and fluid homeostasis. Functionally, the membrane translocation process it mediates, the passive bidirectional diffusion of small inorganic anions, is simpler than the vectorial transport of larger more complex substrates ("allocrites") by most ABC transporters. However, the control of the permeation pathway which cannot go unchecked is necessarily more stringent than in the case of the transporters. There is tight regulation by the phosphorylation/dephosphorylation of the unique CFTR R domain superimposed on the basic $\mathrm{ABC}$ regulation mode of ATP binding and hydrolysis at the dual nucleotide binding sites. As with other ABCC subfamily members, only the second of these sites is hydrolytic in CFTR. The phosphorylation and ATP binding/hydrolysis events do not strongly influence each other; rather, $\mathrm{R}$ domain phosphorylation appears to enable transduction of the nucleotide binding allosteric signal to the responding channel gate. ATP hydrolysis is not required for either the opening or closing gating transitions but efficiently clears the ligand-binding site enabling a new gating cycle to be initiated.
\end{abstract}

Keywords Cystic fibrosis · CFTR · Phosphorylation . ATP binding · ATP hydrolysis

A. A. Aleksandrov $\cdot$ L. A. Aleksandrov $\cdot J$. R. Riordan $(\triangle)$ Department of Biochemistry and Biophysics and Cystic Fibrosis Center, University of North Carolina, Chapel Hill, NC 27599, USA

e-mail: jack_riordan@med.unc.edu

\section{Introduction}

Among the very large number of $\mathrm{ABC}$ proteins now recognized at least at the sequence level, CFTR is distinct as the only one known to be an ion channel. It has not been found in organisms more primitive than elasmobranchs, amphibia, and teleosts where as a chloride channel it plays an important role in salt transport as it does in the respiratory, intestinal, and other epithelial tissues of mammals. Its ion channel function provides the unique opportunity to analyze structure-function relationships of an $\mathrm{ABC}$ protein at the single molecule level. These single channel studies combined with population-based biochemical assays of nucleotide binding and hydrolysis reveal that CFTR acts as a hydrolyzable-ligand-gated channel in which the two processes, binding of the ATP ligand and channel gating are allosterically coupled. Hydrolysis of the ligand is not essential to either channel opening or closing transitions per se but enables repeated cycles of gating to occur.

As an ion channel activated by the binding of a readily available cytoplasmic ligand whose concentration does not undergo large or rapid changes, an additional level of control is required to preclude a continuously active state. This control is provided by the phosphorylation state of the $\mathrm{R}$ domain: transduction of the conformational signal elicited by ATP binding does not occur unless serines in the $\mathrm{R}$ domain are phosphorylated by protein kinase $\mathrm{A}$.

\section{Cystic fibrosis}

$\mathrm{CF}$ is the most frequently occurring genetic disease in Caucasian populations and occurs less frequently in other races. Although disease manifestations reflect environmental factors and modifier genes, the primary cause of the 
disease is mutation of the CFTR gene which codes for an epithelial chloride channel. More than 1,400 different mutations in the CFTR gene have been detected in the screening of patients worldwide. Thus, the major impact of the discovery and study of the gene thus far is the ability to detect mutations in relatives of patients and pre- and postnatally, the latter having become the standard of care in many countries.

Major research efforts are now directed at developing methods to restore CFTR function in patients. Gene replacement therapy efforts have proven extremely challenging from many different perspectives and although progress has been made, significant efficacy has yet to be achieved. A second approach currently being intensively pursued is to overcome the misassembly and misprocessing of the $\Delta$ F508 mutant present in $\sim 90 \%$ of patients. Hence, while several hundred missense mutations that impair specific aspects of CFTR function including channel conductance, gating, and regulation have been identified in patients, a primary focus is on $\Delta \mathrm{F} 508$ because of its prevalence. Deletion of the codon for Phe 508 in NBD1 prevents the polypeptide from achieving a native global conformation [41]. It is polyubiquitinated and degraded by the proteosome at the ER [80]. There is little accumulation of the misassembled polypeptide. Thus, as accurately stated by Cheng, et al. [15] when they first observed this behavior on heterologous expression of $\Delta$ F508 CFTR cDNA, "most cystic fibrosis is caused by CFTR misfolding". Studies of the biosynthetic assembly of wild-type CFTR, although not the subject of this article have helped provide insight into its structure and function [62]. For example, low open probability channel activity occurs in the complete absence of NBD2, indicating that it is not required for channel formation or gating transitions per se (Cui et al., submitted). However, when NBD2 is present either in the full-length protein or when coexpressed with $\triangle \mathrm{NBD} 2$ the ATP hydrolysis, to which it contributes, enables repeated cycles of gating.

\section{CFTR as ion channel and enzyme}

When the CFTR gene was identified by positional cloning $[63,64]$ defective epithelial ion conductance in cystic fibrosis was already known [39, 51]. However, because the sequence of CFTR revealed that it was a member of what came to be known as the ABC family of ATP-dependent membrane transporters, it was not obvious that it coded for the channel responsible for the defective conductance although this possibility was considered [63]. Due to its apparent relationship to transport ATPases, it was postulated to employ a unique mode of gating which is not in thermodynamic equilibrium but is driven by ATP hydrolysis [6]. This would make the CFTR channel distinct from all known ligand- and voltage-gated channels in which ligand binding or an electrical field changes the structural configuration of the close state, allowing thermally driven gating transitions to occur. The idea of ATPase-driven CFTR gating was extended elaborately with proposals that hydrolysis at one NBD controls opening and hydrolysis at the other, closing [21, 34, 82]. Coupling between hydrolysis and gating was suggested to be direct such that individual hydrolytic events could be followed in real time by monitoring channel gating $[8,25]$. These interpretations, requiring an energy transduction pathway based on an unknown physical principle, were made solely on the basis of the effects of different nucleotides on the chloride ion channel activity of wild type and mutant CFTR. Both NBDs were assumed to be sites of ATP hydrolysis similar to the situation in the P-glycoprotein multidrug transporter [70]. We found that only one of the two ATP binding sites in the protein was hydrolytic [4] and this has been incorporated into more up-to-date models of CFTR function [23].

Although the complex regulation of CFTR channel gating is still not fully understood, studies of nucleotide binding and hydrolysis by the protein together with further analysis of single channel gating have provided greater insights (see Table 1 and [62]). An overall assessment of the data leads us to postulate that CFTR is a ligand-gated channel distinguished from other ligand-gated channels only by its ability to hydrolyze its ligand in the binding site and rapidly release the products to complete the cycle. In this view, CFTR ion channel gating occurs by ATP bindinginduced transitions between functionally distinct conformations: open and closed. The foundation of allosteric theory is clear separation of two different processes: ligand binding and structural transitions between different functional states [46]. The molecular details of the allosteric transitions are not well understood but there is no convincing evidence that ATP hydrolysis serves as an energy source for gating. Rather "structural coupling" better describes the relationship between ligand binding and transition to the open-state configuration than the involvement of any chemical transformation including ATP hydrolysis [2-4].

\section{CFTR gating is not dependent on the "energy of ATP hydrolysis"}

This issue is largely due to the use of an antiquated terminology that has no basis in fact. It has long been realized that ATPase-driven mechanical work in molecular 
Table 1 Nucleotide binding and hydrolysis by CFTR

\begin{tabular}{|c|c|c|}
\hline Observations & Interpretation/conclusion & References \\
\hline Photolabeling of CFTR with $\left[\alpha-{ }^{32} \mathrm{P}\right] 8-\mathrm{N}_{3}$ ATP & CFTR binds ATP & [76] \\
\hline NBD2 fusion proteins bind TNP-ATP and hydrolyze ATP & NBD2 has ATPase activity (and adenylate kinase) & {$[55-57]$} \\
\hline NBD1 fusion protein hydrolyzes ATP & CFTR hydrolyses ATP & {$[20,33,41]$} \\
\hline Purified CFTR hydrolyses ATP & CFTR hydrolyses ATP & [45] \\
\hline $\begin{array}{l}{\left[\alpha-{ }^{32} \mathrm{P}\right] 8-\mathrm{N}_{3} \mathrm{ATP} \text { photolabeling vanadate independent at NBD1, }} \\
\text { dependent at NBD2 }\end{array}$ & NBDs have different ATPase activities & [74] \\
\hline Mutation of either Walker A lysine reduces ATPase to very low level & Hydrolysis dependent on two intact NBDs & [54] \\
\hline NBD1 photolabeling stable; NBD2, transient & Nonhydrolyzable AMP-PNP acts on NBD1 & {$[5]$} \\
\hline ATP hydrolysis occurs primarily at NBD2 & $\begin{array}{l}\text { NBD1 is a stable nucleotide binding site, NBD2 a } \\
\text { hydrolytic site }\end{array}$ & {$[4,7]$} \\
\hline CFTR has intrinsic adenylate kinase activity & Adenylate kinase activity supports channel function & {$[25,58]$} \\
\hline
\end{tabular}

Chronology of studies of nucleotide binding and hydrolysis by CFTR or its isolated NBDs.

motors and transport systems does not derive energy from hydrolysis of the $\beta-\gamma$ phosphate chemical bond. Rather, there is a conformation change on relaxation of the strained structure of the catalytic site that has been caused by ATP binding. Thus, there is structural rather than energetic coupling. The energy of a covalent chemical bond may be transformed only to another chemical bond or heat. The chemical bond between $\beta$ and $\gamma$ phosphates of ATP does not have any unique properties. As pointed out by Smith et al. [72] in structural studies of another ABC protein, the energy of ATP binding ultimately drives the overall hydrolytic cycle. This mechanism is analogous to the "binding change" mechanism of ATP synthetase proposed by Boyer [9]. These considerations are important in interpreting the influence of nucleotide interactions with CFTR on channel gating.

Although ATP is employed to do mechanical work in diverse macromolecular systems, this is achieved by the conformational differences between ATP or ADP bound and unbound states of proteins and not from the energy of a chemical bond. In this so-called "entropy engine" the energy of the cleaved covalent chemical bond just sets the upper limit of the elastic energy available from the strained structure. Hydrolysis itself is a power switch rather than a power source. This is a crucial point that distinguishes our understanding from the "demon" mechanism proposed for ABC transporters by Hopfer [30] where information (entropy difference) is derived from the chemical reaction of ATP hydrolysis via an unknown mechanism different from the conventional heat engine. In our interpretation, based on the ability of nonhydrolyzable nucleotide analogs to gate CFTR channels [2], the ligand binding energy is utilized rather than the energy of the chemical bond. Thus, it is unnecessary to invoke an unknown physical phenomenon or "demon".

\section{Is there thermal nonequilibrium in CFTR channel gating?}

Proposals that CFTR gating transitions were not at thermodynamic equilibrium originally stemmed not from experimental data but from the idea that its ATPase must be a device for energy input (see Table 2 for chronology). Gunderson and Kopito [25] reported apparent violation of microscopic reversibility in the transitions between two open states of slightly different conductance that were interpreted as reflecting a gating process not in thermal equilibrium. Because other investigators [6] had already suggested that gating was driven by ATP hydrolysis, Gunderson and Kopito [25] emphasized that this could account for the apparent nonequilibrium in gating. However, one of the two open states was detectable only in strongly filtered records and more detailed analyses revealed that the appearance of a slightly reduced conductance was due only to transitions to a fast flickering mode too rapid for detection of the full conductance state and did not indicate a true second open conductance state [37]. In such a situation, there is no reason to count transitions and refer to the mismatch as evidence of nonequilibrium as most of the transitions have been lost already. More precise kinetic analysis with extended bandwidth does not reveal any evidence of nonequilibrium in CFTR ion channel gating [27]. This confirms our analysis of the temperature dependence of gating transitions which revealed that they were indeed in thermodynamic equilibrium [3].

Although it has been suggested that this conclusion was based on the analysis of different transitions than those used by other investigators [79], this is not the case. In fact, analysis of burst duration as mean open time under conditions where any events faster than $80 \mathrm{~ms}$ were not counted revealed a value of $260 \mathrm{~ms}$ at room temperature [82]. In another 
Table 2 Nucleotide regulation of CFTR channel activity

\begin{tabular}{|c|c|c|}
\hline Observations & Interpretation/conclusion & References \\
\hline Only hydrolyzable nucleotides support channel gating & ATP hydrolysis is necessary for channel gating & {$[6]$} \\
\hline AMP-PNP supports channel activity in presence of $0.5 \mathrm{mM}$ ATP & ATP hydrolysis not essential for gating & {$[53]$} \\
\hline Vanadate does not inhibit channel opening but inhibits closing & Free energy of hydrolysis drives gating & {$[8]$} \\
\hline $\begin{array}{l}\text { AMP-PNP does not support opening but inhibits closing at room } \\
\text { temperature }\end{array}$ & $\begin{array}{l}\text { ATP hydrolysis at one NBD controls opening, the } \\
\text { other, closing }\end{array}$ & {$[35]$} \\
\hline ATP activation of gating noncooperative & Activation by nucleotide occurs at a single site & {$[78]$} \\
\hline $\begin{array}{l}\text { ATP-dependent gating not influenced by poorly hydrolyzable } \\
\text { analogues at }+37^{\circ} \mathrm{C}\end{array}$ & Binding site sensitive to phosphate chain configuration & [69] \\
\hline $\begin{array}{l}\text { NBD1 mutations reduce frequency of openings, NBD2 mutations } \\
\text { prolong open state }\end{array}$ & $\begin{array}{l}\text { ATP hydrolysis at NBD1 initiates activity; at NBD2 } \\
\text { terminates }\end{array}$ & {$[11]$} \\
\hline $\mathrm{Q}_{10}$ opening $=9.6, \mathrm{Q}_{10}$ closing $=3.6$ & Opening more temp sensitive than closing & {$[46]$} \\
\hline $\begin{array}{l}2 \text { apparent open conductance states and NBD2 mutations prolong } \\
\text { opening }\end{array}$ & $\begin{array}{l}\text { Gating not in thermal equilibrium and NBD2 main } \\
\text { controller of gating }\end{array}$ & {$[26]$} \\
\hline $\mathrm{E}_{\mathrm{a}}$ opening $\sim 100 \mathrm{~kJ} / \mathrm{mol}$, closing $\sim 10 \mathrm{~kJ} / \mathrm{mol}$ & Gating is in thermal equilibrium & [3] \\
\hline ATP minus divalent cation or AMP-PNP support gating & Hydrolysis is not essential for gating & {$[2,68]$} \\
\hline Nonhydrolyzable nucleotides support opening and prolong closing & Hydrolysis is not essential for gating & {$[80]$} \\
\hline NBD2 hydrolytic base mutant (E1371) locks channel in open state & Hydrolysis accelerates closing & [79] \\
\hline $\begin{array}{l}\text { Functionally compensatory mutations in NBD1 signature and NBD2 } \\
\text { Walker A }\end{array}$ & $\begin{array}{l}\text { NBD dimerization provides transition state for } \\
\text { channel opening }\end{array}$ & {$[79]$} \\
\hline
\end{tabular}

Chronology of studies of the nucleotide-dependence of CFTR chloride channel activity.

laboratory, burst analysis with extended bandwidth revealed a burst duration of $267 \mathrm{~ms}$ at room temperature [49]. In both these cases, the values are closer to the value of $220 \mathrm{~ms}$ we reported as a mean open time [3] than to the values, determined at room temperature, of $1,210 \mathrm{~ms}$ by Mathews et al. [45] or $896 \mathrm{~ms}$ by Csanady et al. [17]. It is interesting to note that in both the latter papers, high PKA concentrations of 200-300 nM were used (compare with $100 \mathrm{U} / \mathrm{ml}=45 \mathrm{nM}$ PKA accompanied by $0.3 \mathrm{mM}$ phosphate used by Aleksandrov and Riordan [3]. The burst duration returned to the common value of $260 \mathrm{~ms}$ after PKA wash out [17]. One might speculate that these differences could relate to the high phosphate concentration typically used in the stock PKA buffer solution and known to affect CFTR ion channel gating. Importantly, our mean open and closed times when determined at $35^{\circ} \mathrm{C}$ rather than room temperature were virtually identical to those of Carson et al. [11] and similar to those of Mathews et al. [45]. As a result, the slope of the Arrhenius plot of the closing rate observed by Aleksandrov and Riordan [3] would be underestimated by $15 \%$, but even with this taken into account, the slope is much less steep than that of Mathews et al. [45]. Thus, there does not appear to be any published data which legitimately disputes the demonstration of thermal equilibrium between the open and closed states of the CFTR channel.

Furthermore, there is no contradiction between the irreversible nature of ATP hydrolysis and the thermal equilibrium of the channel gating. As mentioned above, there are two different processes typically considered in allosteric theory. In our case, one of them is ligand binding that is the signal formation process while the other is the transition between open and closed conformations or the functional response to the signal. In single channel recordings, we have the opportunity to measure microscopic parameters of only the second process. The principle of microscopic reversibility requires that under equilibrium conditions, the transition frequency in both directions should be the same. For reversibility of the gating process, this means:

$P_{O} * k_{C}=P_{C} * k_{O}$,

where $P_{\mathrm{o}}=$ the probability of the open state; $P_{\mathrm{c}}=1-P_{\mathrm{o}}$, the probability of the closed state; $k_{\mathrm{c}}=1 / \tau_{\mathrm{o}}$, rate constant of channel closing; $k_{\mathrm{o}}=1 / \tau_{\mathrm{c}}$, rate constant of channel opening. The above relationship holds if $P_{O}=\tau_{O} /\left(\tau_{O}+\tau_{C}\right)$. The experimental data met this requirement and thereby enabled us to consider CFTR ion channel gating as a reversible thermally driven process [3]. The clear separation of two different processes is an essential element of allosteric theory. Ligand binding and hydrolysis are separate and distinct processes from gating and irreversibility is not crucial to the relationship between the two processes as long as the probability of the ligand bound state is constant in steady state as well as at equilibrium. 


\section{ATP hydrolysis is not essential for either channel opening or closing}

Although it had been claimed that ATP hydrolysis is essential for channel opening [22, 71], there is now ample evidence this is not the case $[2,36,79]$. It is interesting to note that the evidence for a nonhydrolytic mode of CFTR channel gating comes from experiments using the same "nonhydrolytic conditions", i.e., nonhydrolyzable analogues and divalent cation-free solutions, as were earlier employed to argue the opposite and in at least two cases by the same laboratories [79], emphasizing that opening is slower under nonhydrolytic than hydrolytic conditions. However, this kinetic difference does not change the fact that gating occurs in the absence of hydrolysis and hence is not dependent upon it. Diminution by an order of magnitude in opening frequency in any case would reflect only a $2.3 \mathrm{RT}$ or $\sim 5.8 \mathrm{~kJ} / \mathrm{mol}$ change in the energetic barrier to be overcome.

\section{CFTR is a hydrolyzable-ligand-gated channel}

Because ATP hydrolysis appears not to be essential for either CFTR channel opening or closing, what is the role of ATP hydrolysis by the protein? We postulate that it provides an efficient means of getting rid of the ligand whose binding is responsible for the initiation of gating. The closest analogy would be with cyclic nucleotide-activated channels where the ligands are also cytoplasmic nucleotides [38]. These behave as classic ligand-gated channels, even though they have adopted a voltage-gated channel structural motif, responding to the binding and dissociation of the ligand depending on its bulk concentration. Regulation is at the level of these concentrations determined by the activities of cyclases and phosphodiesterases which are in turn controlled by upstream signals. Cellular ATP is not regulated in this acute fashion so that drastic decreases in concentration required for its dissociation from binding sites is not a sufficient means of terminating its impact. Hydrolysis at the second ATP binding site of CFTR provides this efficiency. While dissociation of unhydrolyzed ATP can allow termination of gating under nonhydrolytic conditions, ADP produced under hydrolytic conditions dissociates much more rapidly [1]. In this view, the novel feature of CFTR as a ligand-gated channel is its ability to change the ligand chemical structure to promote dissociation from the binding site. This interpretation is consistent with the fact that specificity of binding and activation by the ligand is primarily in the geometry of phosphate moiety; there is a very strong preference for purine nucleoside triphosphates that can be hydrolyzed at the site responsible for channel activation. Changes in bond angles in the phosphate chain result in less binding and less effective activation.
These considerations of available data lead to the simplified hypothetical coupling between ATP binding and hydrolysis and CFTR ion channel gating that is shown in Fig. 1. This type of diagram was originally employed by Hille to display allosteric regulation between microscopic states of nACh receptor and ion channel gating in real time [28] Interconversion between microscopic states of the nucleotide binding sites are shown in Fig. 1, where CFTR $(\mathrm{X}, \mathrm{X})$ denotes the binding states of the two sites. The activated state CFTR*(ATP,ATP) is one with structural coupling between the binding sites and the channel open state that is available when the $\mathrm{R}$ domain is phosphorylated. The probability of the channel open state correlates with the probability of the CFTR*(ATP,ATP) state of the binding sites that is constant under steady-state conditions. Hence, reversibility of the interconvertions between the different CFTR $(X, X)$ species is not required to establish reversibility of the gating process. Moreover, on this basis, one can ignore the multiplicity of structural species coupled with the channel closed state and reduce the gating kinetic scheme to reversible thermally driven transitions between closed and open states [3].

To underline the ability of CFTR to bind and hydrolyze ATP independently of channel gating status, we place both CFTR (ATP,ATP) and CFTR*(ATP,ATP) states in a common box connected to CFTR (ATP,ADP) by the same pathway. In the hypothetical time course of gating shown in the upper part of Fig. 1, the initial configuration corre-

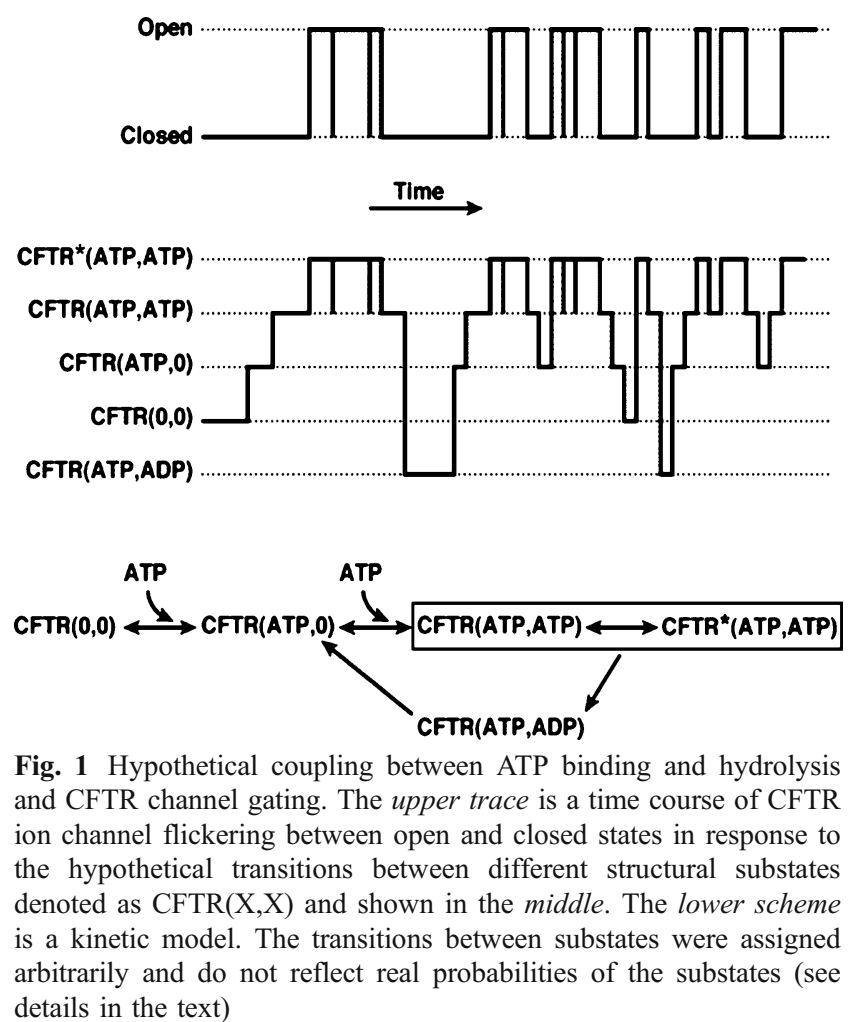


sponds to the closed state before both nucleotide binding sites bind MgATP followed by the fitting of their structures to the tightly bound ligand which sends a conformational signal for channel opening through a pathway involving the phosphorylated $\mathrm{R}$ domain. The channel adopts the open state after the CFTR*(ATP,ATP) structure becomes available. The burst-like behavior is a result of short-lived structural disruption in the signal transduction pathway. After ATP hydrolysis at site II, the channel switches to a long-lived closed state. The channel remains closed until a new ATP molecule is bound at that site restoring the activated CFTR*(ATP,ATP) structure. This is the so-called hydrolytic pathway.

The long-lived closed state may result not only from ATP hydrolysis but also from incorrect orientation of the ligand in the binding site or its complete dissociation as well as from disruption of the signal transduction pathway. This was observed with nonhydrolyzable analogs and termed the nonhydrolytic pathway [2]. Only two hydrolytic closings of a total of six burst terminations are shown in the Fig. 1. While only a hypothetical example of the loose correlation between burst termination and ATP hydrolysis, it provides a reasonable way to explain all available experimental data.

On the basis of these and other considerations, CFTR can be classified as a ligand-gated channel. Its only peculiarity as such is its inherent ability to rid itself of the ligand. The hydrolytic transformation of the ligand in at least one of its binding sites provides an even more effective termination than a reduction in bulk ligand concentration which is required in the case of other ligand gated channels.

\section{Role of the "nucleotide-sandwich"}

At low resolution, the 3D structure of CFTR is similar to that of other $\mathrm{ABC}$ proteins that are membrane transporters rather than ion channels [65]. The high-resolution structure of the first nucleotide-binding domain $[42,43]$ indicates that it shares the same fold as NBDs of other ABC proteins. NBD1 and NBD2 are quite dissimilar at the sequence and functional levels and the structure of NBD2 has not yet been determined. However, important advances in the understanding of other members of the large $\mathrm{ABC}$ family in the past few years have indicated that their two NBDs closely associate forming two composite ATP binding sites at the interface between them rather than each NBD forming a separate site [47, 72]. CFTR site I is formed primarily by the Walker A sequence motif of NBD1 and $\mathrm{ABC}$ signature motif of NBD2 and is a nonhydrolytic stable ATP binding site, whereas site II formed by the Walker A motif of NBD2 and the signature of NBD1 hydrolyses the ATP bound there [4, 7]. This asymmetry in the NBDs apparently is not specifically related to the ion channel function of CFTR as it also occurs in other members of the human ABCC subfamily which are transporters.

Conformational movements within and between the two NBDs when ATP is bound and hydrolyzed probably contribute to the transport function of $\mathrm{ABC}$ transporters [31], and it has recently been suggested that these movements contribute to CFTR channel opening [78]. These investigators observed compensatory influences of mutations of a specific pair of resides in the signature sequence of NBD1 and the Walker A motif of NBD2 and concluded that ATP-mediated dimerization of the two domains promotes the transition state for channel opening. It does seem very likely that ATP binding at site II of CFTR may tighten the association between the domains already fostered by the stably bound nucleotide at site I.

However, the particular application of so-called double mutant-cycle analysis, which Vergani et al. [78] employed using gating rate constants to evaluate activation energies for the overall gating process, may be questioned. The basic assumptions established for Eyring rate theory are not valid for such a multistep process. Dimer formation may be one of several configuration changes which occurs in the closed state but the relation of the activation energy of dimer formation and the effective $E_{\mathrm{a}}$ derived from the rate constant of channel opening is not known. In addition to these theoretical concerns, it is noted that the effect of the NBD1 signature mutation, R555K on the rate of opening is quite different from that reported previously by Teem et al. [74] and as measured in our laboratory. In both latter cases, the mutation significantly reduced the channel closing rate without influencing the opening rate whereas Vergani et al. [78] observe just the opposite, with this slowing of opening used as the foundation of their entire analysis. Furthermore, gating transitions are clearly not directly controlled by NBD dimer formation and dissociation because they occur, albeit at low frequency, in the complete absence of NBD2 (Cui et al., submitted). Thus, while a generally common molecular mechanism involving ATP interactions at their binding sites at the interface between NBDs may modulate the transmembrane permeability pathways in $\mathrm{ABC}$ transporters and the CFTR anion channel, the specific intramolecular steps involved remain to be elucidated.

\section{CFTR control by phosphorylation}

Apparently unique to CFTR among $\mathrm{ABC}$ proteins, there is an additional level of control by phosphorylation and dephosphorylation which enables it to be dynamically regulated by conditions that change levels of cellular cAMP and hence PKA activity [16, 22, 69]. Modulation by 
phosphorylation which is common to many other ion channels, both ligand- and voltage-gated, is rather absolute in the case of CFTR [22] and may be required for propagation of the conformational impact of ligand binding to the pore. Patch-clamp studies with CF epithelial cells in the 1980s before the discovery of CFTR indicated a defective cAMP-stimulated anion current $[35,66]$ and the CFTR sequence later revealed the presence of a large cluster of consensus sites for phosphorylation by cAMPdependent protein kinase $\mathrm{A}$ in the $\mathrm{R}$ domain [63]. Extensive studies have confirmed that the phosphorylation state of these sites strictly controls the activity of the CFTR chloride channel $[12,48]$. This control is superimposed on the regulation of channel gating by ATP binding and hydrolysis at the NBDs $[3,6]$ and is essential to keep the channel from gating continuously. When the chloride permeation pathway is not required for salt secretion or reabsorption, phosphatase activity maintains CFTR in an unphosphorylated and inactive state [26]. When chloride permeability is required, agonists that increase cAMP elevate PKA activity which phosphorylates $\mathrm{R}$ domain sites enabling ATP-dependent channel gating.

This stringent control by phosphorylation/dephosphorylation is absolutely necessary to the utilization of the ABC transporter structural architecture as an ion channel. The transporters utilize available cytoplasmic ATP to transport their substrates depending on availability and do not require further control. Ion channels, on the other hand, cannot serve useful functions unless their gating can be turned on and off. This is achieved either by conformational responses to changes in membrane potential (voltage-gated) or ligand binding (ligand-gated). In general, ligand-gated channels sense changes in ligand concentrations whereas the cytoplasmic concentration of the CFTR-activating ligand, ATP remains relatively constant within the low millimolar range. Therefore, without an additional level of control, the CFTR channel would always be active. The R domain which appears to be unique not only within the large $\mathrm{ABC}$ protein family but among all sequences known thus far provides a "phosphorylation control module".

The fact that this module contains a string of sites for phosphorylation by PKA that is highly conserved among species would seem to emphasize their importance to the molecule's function. The mechanism whereby gating is enabled when the $\mathrm{R}$ domain is phosphorylated and not when it is unphosphorylated is not well understood despite extensive investigations $[45,76]$. Both the introduction of negative charges by the phosphoryl groups [61] and conformation changes [20] play some role. The unphosphorylated $\mathrm{R}$ domain has an inhibitory action that is relieved on phosphorylation but the phosphorylated domain has been reported to also have a positive stimulating effect [48]. Interpretation of how the multiple sites are employed in permitting channel gating remains far from clear. Mutagenesis of sites individually and in sets has provided certain insights, with removal of some sites having greater impact than others $[12,16]$. We found it necessary to remove a total of 15 sites to render the channel unresponsive to PKA (70). At least two of the sites have been reported to have inhibitory effects on channel activity [18, $76,81]$.

Protein kinase $\mathrm{C}$ also plays a role in CFTR activation [13], primarily in the case of the human protein, by enhancing the response to PKA [14]. This seems to occur without elevating or accelerating phosphorylation by PKA [14]. Strikingly, introduction of a particularly significant $R$ domain PKC site of Xenopus CFTR into the human channel rendered it directly responsive to activation by PKC alone when expressed in Xenopus oocytes [10]. All mechanistic studies of CFTR activation by phosphorylation have necessarily been done in model heterologous expression systems and it is assumed that similar mechanisms apply in situ in tissues where CFTR function is crucial. CFTR activation in the intestine and airways is elicited by agonists that increase cellular cyclic nucleotides and in excised membrane patches in cell lines derived from these tissues by direct application of PKA [48].

From this large overall body of evidence, it would appear that CFTR evolved to be controlled primarily by phosphorylation/dephosphorylation in response to humoral agonists acting through cyclic nucleotides and their dependent kinases. It must be pointed out that there also are reports of CFTR activation by nonphosphorylationdependent mechanisms. In one of the most physiologically relevant experimental systems, the perfused isolated sweat duct, GTP $\gamma \mathrm{S}$-stimulated chloride conductance leading to the suggestion that heterotrimeric $\mathrm{G}$ proteins may be able to directly activate CFTR [58, 60]. Although this activation was blocked by the general protein kinase inhibitor, staurosporine, it was thought not to be due to PKA because there was no accumulation of cAMP in these GTP $\gamma \mathrm{S}$ stimulated permeabilized ducts nor did adenylate cyclase inhibitors prevent the activation. However, the stimulatory effects of added cAMP and GTP $\gamma \mathrm{S}$ were not additive indicating that they were convergent at some point. While several $G$ proteins localized to the apical membrane of the sweat duct cells, it was not clear which might be involved. In the apical membrane of airway epithelial cells $G_{s}$ couples activated adenosine $\mathrm{A} 2 \mathrm{~b}$ receptors to adenylate cyclase producing cAMP to stimulate PKA which phosphorylates and activates CFTR [33]. In the same cells, $\mathrm{P} 2 \mathrm{Y}_{2}$ purinergic receptors coupled to phospholipase $C$ via $G_{q}$ may cause some activation of CFTR via a calcium-independent PKC activated by the diacyl glycerol released [50]. However, because only slight CFTR activation occurs solely due to PKC [14], it is not certain if the purinergic activation occurs 
entirely independently of PKA. Also, although both of these experimental systems, the sweat ducts and the airway cells, are of the utmost relevance to cystic fibrosis, the measurements of transepithelial currents are quite indirect measures of CFTR. Other reports of PKA-independent CFTR activation include the effects of dATP [4], PIP $_{2}$ [29], and glutamate [59]. Although of interest mechanistically, the dATP effect is almost certainly nonphysiological while changes in cellular $\mathrm{PIP}_{2}$ concentrations do occur. In none of these latter cases were measurements of the phosphorylation state of the protein made. It is possible that some of the important R domain PKA sites may have become phosphorylated due to inhibition of a phosphatase that normally maintains the unphosphorylated state.

\section{Conclusion}

CFTR provides a novel intersection of the ion channel and $\mathrm{ABC}$ transporter fields, faithfully obeying the principals while expanding the horizons of each.

Acknowledgements Research results from our laboratory discussed in this report were supported by the NIH and CFF.

\section{References}

1. Aleksandrov AA, Aleksandrov L, Riordan JR (2002) Nucleoside triphosphate pentose ring impact on CFTR gating and hydrolysis. FEBS Lett 518:183-188

2. Aleksandrov AA, Chang X, Aleksandrov L, Riordan JR (2000) The non-hydrolytic pathway of cystic fibrosis transmembrane conductance regulator ion channel gating. J Physiol 528(Pt 2):259-265

3. Aleksandrov AA, Riordan JR (1998) Regulation of CFTR ion channel gating by MgATP. FEBS Lett 431:97-101

4. Aleksandrov L, Aleksandrov AA, Chang XB, Riordan JR (2002) The first nucleotide binding domain of cystic fibrosis transmembrane conductance regulator is a site of stable nucleotide interaction, whereas the second is a site of rapid turnover. J Biol Chem 277:15419-15425

5. Aleksandrov L, Mengos A, Chang X, Aleksandrov A, Riordan JR (2001) Differential interactions of nucleotides at the two nucleotide binding domains of the cystic fibrosis transmembrane conductance regulator. J Biol Chem 276:12918-12923

6. Anderson MP, Berger HA, Rich DP, Gregory RJ, Smith AE, Welsh MJ (1991) Nucleoside triphosphates are required to open the CFTR chloride channel. Cell 67:775-784

7. Basso C, Vergani P, Nairn AC, Gadsby DC (2003) Prolonged nonhydrolytic interaction of nucleotide with CFTR's NH2terminal nucleotide binding domain and its role in channel gating. J Gen Physiol 122:333-348

8. Baukrowitz T, Hwang TC, Nairn AC, Gadsby DC (1994) Coupling of CFTR Cl-channel gating to an ATP hydrolysis cycle. Neuron 12:473-482

9. Boyer PD (1998) Energy, life and ATP. Angew Chem Int Ed $37: 2296-2307$
10. Button B, Reuss L, Altenberg GA (2001) PKC-mediated stimulation of amphibian CFTR depends on a single phosphorylation consensus site. Insertion of this site confers PKC sensitivity to human CFTR. J Gen Physiol 117:457-468

11. Carson MR, Travis SM, Welsh MJ (1995) The two nucleotidebinding domains of cystic fibrosis transmembrane conductance regulator (CFTR) have distinct functions in controlling channel activity. J Biol Chem 270:1711-1717

12. Chang XB, Tabcharani JA, Hou YX, Jensen TJ, Kartner N, Alon N, Hanrahan JW, Riordan JR (1993) Protein kinase A (PKA) still activates CFTR chloride channel after mutagenesis of all 10 PKA consensus phosphorylation sites. J Biol Chem 268:11304-11311

13. Chappe V, Hinkson DA, Howell LD, Evagelidis A, Liao J, Chang XB, Riordan JR, Hanrahan JW (2004) Stimulatory and inhibitory protein kinase $\mathrm{C}$ consensus sequences regulate the cystic fibrosis transmembrane conductance regulator. Proc Natl Acad Sci USA 101:390-395

14. Chappe V, Hinkson DA, Zhu T, Chang XB, Riordan JR, Hanrahan JW (2003) Phosphorylation of protein kinase C sites in NBD1 and the R domain control CFTR channel activation by PKA. J Physiol 548:39-52

15. Cheng SH, Gregory RJ, Marshall J, Paul S, Souza DW, White GA, O'Riordan CR, Smith AE (1990) Defective intracellular transport and processing of CFTR is the molecular basis of most cystic fibrosis. Cell 63:827-834

16. Cheng SH, Rich DP, Marshall J, Gregory RJ, Welsh MJ, Smith AE (1991) Phosphorylation of the R domain by cAMP-dependent protein kinase regulates the CFTR chloride channel. Cell 66:1027-1036

17. Csanady L, Chan KW, Seto-Young D, Kopsco DC, Nairn AC, Gadsby DC (2000) Severed channels probe regulation of gating of cystic fibrosis transmembrane conductance regulator by its cytoplasmic domains. J Gen Physiol 116:477-500

18. Csanady L, Seto-Young D, Chan KW, Cenciarelli C, Angel BB, Qin J, McLachlin DT, Krutchinsky AN, Chait BT, Nairn AC, Gadsby DC (2005) Preferential phosphorylation of R-domain Serine 768 dampens activation of CFTR channels by PKA. J Gen Physiol 125:171-186

19. Duffieux F, Annereau JP, Boucher J, Miclet E, Pamlard O, Schneider M, Stoven V, Lallemand JY (2000) Nucleotide-binding domain 1 of cystic fibrosis transmembrane conductance regulator production of a suitable protein for structural studies. Eur J Biochem 267:5306-5312

20. Dulhanty AM, Riordan JR (1994) Phosphorylation by cAMPdependent protein kinase causes a conformational change in the $\mathrm{R}$ domain of the cystic fibrosis transmembrane conductance regulator. Biochemistry 33:4072-4079

21. Gadsby DC, Nairn AC (1994) Regulation of CFTR channel gating. Trends Biochem Sci 19:513-518

22. Gadsby DC, Nairn AC (1999) Control of CFTR channel gating by phosphorylation and nucleotide hydrolysis. Physiol Rev 79:S77S107

23. Gadsby DC, Vergani P, Csanady L (2006) The ABC protein turned chloride channel whose failure causes cystic fibrosis. Nature 440:477-483

24. Gross $\mathrm{CH}$, Abdul-Manan N, Fulghum J, Lippke J, Liu X, Prabhakar P, Brennan D, Willis MS, Faerman C, Connelly P, Raybuck S, Moore J (2006) Nucleotide-binding domains of cystic fibrosis transmembrane conductance regulator, an $\mathrm{ABC}$ transporter, catalyze adenylate kinase activity but not ATP hydrolysis. J Biol Chem 281:4058-4068

25. Gunderson KL, Kopito RR (1995) Conformational states of CFTR associated with channel gating: the role of ATP binding and hydrolysis. Cell 82:231-239

26. Hanrahan JH, Gentzsch M, Riordan JR (2003) The cystic fibrosis transmembrane conductance regulator (ABCC7). In: Holland B, 
Higgins CF, Kuchler K, Cole SPC (eds) ABC Proteins: from bacteria to man. Elsevier, New York, pp 589-618

27. Hennager DJ, Ikuma M, Hoshi T, Welsh MJ (2001) A conditional probability analysis of cystic fibrosis transmembrane conductance regulator gating indicates that ATP has multiple effects during the gating cycle. Proc Natl Acad Sci USA 98:3594-3599

28. Hille B (2001) Ligand-gated channels of fast chemical synapses. In: Hille B (ed) Ion channels of excitable membranes, 3rd edn. Sinauer Associates, Inc., USA, pp 84-184

29. Himmel B, Nagel G (2004) Protein kinase-independent activation of CFTR by phosphatidylinositol phosphates. EMBO Rep 5:85-90

30. Hopfer U (2002) A Maxwell's demon type of membrane transport: possibility for active transport by $\mathrm{ABC}$-type transporters? J Theor Biol 214:539-547

31. Horn C, Bremer E, Schmitt L (2003) Nucleotide dependent monomer/dimer equilibrium of OpuAA, the nucleotide-binding protein of the osmotically regulated $\mathrm{ABC}$ transporter OpuA from bacillus subtilis. J Mol Biol 334:403-419

32. Howell LD, Borchardt R, Cohn JA (2000) ATP hydrolysis by a CFTR domain: pharmacology and effects of G551D mutation. Biochem Biophys Res Commun 271:518-525

33. Huang P, Lazarowski ER, Tarran R, Milgram SL, Boucher RC, Stutts MJ (2001) Compartmentalized autocrine signaling to cystic fibrosis transmembrane conductance regulator at the apical membrane of airway epithelial cells. Proc Natl Acad Sci USA 98:14120-14125

34. Hwang T-C, Baukrowtiz G, Nagel G, Horie AC, Gadsby DC (1994) Regulation of gating of cystic fibrosis transmembrane conductance regulator $\mathrm{C} 1$ channels by phosphorylation and ATP hydrolysis. Proc Natl Acad Sci USA 91:4698-4702

35. Hwang TC, Lu L, Zeitlin PL, Gruenert DC, Huganir R, Guggino WB (1989) Cl-channels in CF: lack of activation by protein kinase $\mathrm{C}$ and cAMP-dependent protein kinase. Science 244:1351-1356

36. Ikuma M, Welsh MJ (2000) Regulation of CFTR Cl- channel gating by ATP binding and hydrolysis. Proc Natl Acad Sci USA 97:8675-8680

37. Ishihara H, Welsh MJ (1997) Block by MOPS reveals a conformation change in the CFTR pore produced by ATP hydrolysis. Am J Physiol 273:C1278-C1289

38. Kaupp UB, Seifert R (2002) Cyclic nucleotide-gated ion channels. Physiol Rev 82:769-824

39. Knowles MR, Stutts MJ, Spock A, Fischer N, Gatzy JT, Boucher RC (1983) Abnormal ion permeation through cystic fibrosis respiratory epithelium. Science 221:1067-1070

40. Ko YH, Pedersen PL (1995) The first nucleotide binding fold of the cystic fibrosis transmembrane conductance regulator can function as an active ATPase. J Biol Chem 270:22093-22096

41. Kopito RR (1999) Biosynthesis and degradation of CFTR. Physiol Rev 79:S167-S173

42. Lewis HA, Buchanan SG, Burley SK, Conners K, Dickey M, Dorwart M, Fowler R, Gao X, Guggino WB, Hendrickson WA, Hunt JF, Kearins MC, Lorimer D, Maloney PC, Post KW, Rajashankar KR, Rutter ME, Sauder JM, Shriver S, Thibodeau PH, Thomas PJ, Zhang M, Zhao X, Emtage S (2004) Structure of nucleotide-binding domain 1 of the cystic fibrosis transmembrane conductance regulator. EMBO J 23:282-293

43. Lewis HA, Zhao X, Wang C, Sauder JM, Rooney I, Noland BW, Lorimer D, Kearins MC, Conners K, Condon B, Maloney PC, Guggino WB, Hunt JF, Emtage S (2005) Impact of the deltaF508 mutation in first nucleotide-binding domain of human cystic fibrosis transmembrane conductance regulator on domain folding and structure. J Biol Chem 280:1346-1353

44. Li C, Ramjeesingh M, Wang W, Garami E, Hewryk M, Lee D, Rommens JM, Galley K, Bear CE (1996) ATPase activity of the cystic fibrosis transmembrane conductance regulator. J Biol Chem 271:28463-28468
45. Mathews CJ, Tabcharani JA, Hanrahan JW (1998) The CFTR chloride channel: nucleotide interactions and temperature-dependent gating. J Membr Biol 163:55-66

46. Monod J, Wyman J, Changeux J-P (1965) On the nature of allosteric transitions: a plausible model. J Mol Biol 12:88-118

47. Moody JE, Millen L, Binns D, Hunt JF, Thomas PJ (2002) Cooperative, ATP-dependent association of the nucleotide binding cassettes during the catalytic cycle of ATP-binding cassette transporters. J Biol Chem 277:21111-21114

48. Ostedgaard LS, Baldursson O, Welsh MJ (2001) Regulation of the cystic fibrosis transmembrane conductance regulator $\mathrm{Cl}$ - channel by its R domain. J Biol Chem 276:7689-7692

49. Ostedgaard LS, Randak C, Rokhlina T, Karp P, Vermeer D, Ashbourne Excoffon KJ, Welsh MJ (2003) Effects of C-terminal deletions on cystic fibrosis transmembrane conductance regulator function in cystic fibrosis airway epithelia. Proc Natl Acad Sci USA 100:1937-1942

50. Paradiso AM, Ribeiro CM, Boucher RC (2001) Polarized signaling via purinoceptors in normal and cystic fibrosis airway epithelia. J Gen Physiol 117:53-67

51. Quinton PM (1983) Chloride impermeability in cystic fibrosis. Nature 301:421-422

52. Quinton PM, Reddy MM (1992) Control of CFTR chloride conductance by ATP levels through non-hydrolytic binding. Nature 360:79-81

53. Ramjeesingh M, Li C, Garami E, Huan LJ, Galley K, Wang Y, Bear CE (1999) Walker mutations reveal loose relationship between catalytic and channel-gating activities of purified CFTR (cystic fibrosis transmembrane conductance regulator). Biochemistry 38:1463-1468

54. Randak C, Neth P, Auerswald EA, Assfalg-Machleidt I, Roscher AA, Hadorn HB, Machleidt W (1996) A recombinant polypeptide model of the second predicted nucleotide binding fold of the cystic fibrosis transmembrane conductance regulator is a GTPbinding protein. FEBS Lett 398:97-100

55. Randak C, Neth P, Auerswald EA, Eckerskorn C, AssfalgMachleidt I, Machleidt W (1997) A recombinant polypeptide model of the second nucleotide-binding fold of the cystic fibrosis transmembrane conductance regulator functions as an active ATPase, GTPase and adenylate kinase. FEBS Lett 410: 180-186

56. Randak C, Roscher AA, Hadorn HB, Assfalg-Machleidt I, Auerswald EA, Machleidt W (1995) Expression and functional properties of the second predicted nucleotide binding fold of CFTR fused to GST. FEBS Lett 363:189-194

57. Randak CO, Welsh MJ (2005) ADP inhibits function of the ABC transporter cystic fibrosis transmembrane conductance regulator via its adenylate kinase activity. Proc Natl Acad Sci USA 102:2216-2220

58. Reddy MM, Quinton PM (2001) cAMP-independent phosphorylation activation of CFTR by $\mathrm{G}$ proteins in native human sweat duct. Am J Physiol Cell Physiol 280:C604-C613

59. Reddy MM, Quinton PM (2003) Control of dynamic CFTR selectivity by glutamate and ATP in epithelial cells. Nature 423:756-760

60. Reddy MM, Sun D, Quinton PM (2001) Apical heterotrimeric g-proteins activate CFTR in the native sweat duct. J Membr Biol 179:51-61

61. Rich DP, Berger HA, Cheng SH, Travis SM, Saxena M, Smith AE, Welsh MJ (1993) Regulation of the cystic fibrosis transmembrane conductance regulator $\mathrm{Cl}$ - channel by negative charge in the R domain. J Biol Chem 268:20259-20267

62. Riordan JR (2005) Assembly of functional CFTR chloride channels. Annu Rev Physiol 67:701-718

63. Riordan JR, Rommens JM, Kerem B, Alon N, Rozmahel R, Grzelczak Z, Zielenski J, Lok S, Plavsic N, Chou JL et al (1989) 
Identification of the cystic fibrosis gene: cloning and characterization of complementary DNA. Science 245:1066-1073

64. Rommens JM, Iannuzzi MC, Kerem B-S, Drumm ML, Melmer G, Dean M, Rozmahel R, Cole JL, Kennedy D, Hidaka N, Zsiga M, Buchwald M, Riordan JR, Tsui L-C, Collins F (1989) Identification of the cystic fibrosis gene: chromosome walking and jumping. Science 245:1059-1065

65. Rosenberg MF, Kamis AB, Aleksandrov LA, Ford RC, Riordan JR (2004) Purification and crystallization of the cystic fibrosis transmembrane conductance regulator (CFTR). J Biol Chem 279:39051-39057

66. Schoumacher RA, Shoemaker RL, Halm DR, Tallant EA, Wallace RW, Frizzell RA (1987) Phosphorylation fails to activate chloride channels from cystic fibrosis airway cells. Nature 330:752-754

67. Schultz BD, Bridges RJ, Frizzell RA (1996) Lack of conventional ATPase properties in CFTR chloride channel gating. J Membr Biol 151:63-75

68. Schultz BD, Venglarik CJ, Bridges RJ, Frizzell RA (1995) Regulation of CFTR $\mathrm{Cl}-$ channel gating by ADP and ATP analogues. J Gen Physiol 105:329-361

69. Seibert FS, Chang XB, Aleksandrov AA, Clarke DM, Hanrahan JW, Riordan JR (1999) Influence of phosphorylation by protein kinase A on CFTR at the cell surface and endoplasmic reticulum. Biochim Biophys Acta 1461:275-283

70. Senior AE, Gadsby DC (1997) ATP hydrolysis cycles and mechanism in P-glycoprotein and CFTR. Semin Cancer Biol $8: 143-150$

71. Sheppard DN, Welsh MJ (1999) Structure and function of the CFTR chloride channel. Physiol Rev 79:S23-S45

72. Smith PC, Karpowich N, Millen L, Moody JE, Rosen J, Thomas PJ, Hunt JF (2002) ATP binding to the motor domain from an $\mathrm{ABC}$ transporter drives formation of a nucleotide sandwich dimer. Mol Cell 10:139-149
73. Szabo K, Szakacs G, Hegeds T, Sarkadi B (1999) Nucleotide occlusion in the human cystic fibrosis transmembrane conductance regulator. Different patterns in the two nucleotide binding domains. J Biol Chem 274:12209-12212

74. Teem JL, Carson MR, Welsh MJ (1996) Mutation of R555 in CFTR-delta F508 enhances function and partially corrects defective processing. Recept Channels 4:63-72

75. Travis SM, Carson MR, Ries DR, Welsh MJ (1993) Interaction of nucleotides with membrane-associated cystic fibrosis transmembrane conductance regulator. J Biol Chem 268:15336-15339

76. Vais H, Zhang R, Reenstra WW (2004) Dibasic phosphorylation sites in the R domain of CFTR have stimulatory and inhibitory effects on channel activation. Am J Physiol Cell Physiol 287: C737-C745

77. Venglarik CJ, Schultz BD, Frizzell RA, Bridges RJ (1994) ATP alters current fluctuations of cystic fibrosis transmembrane conductance regulator: evidence for a three-state activation mechanism. J Gen Physiol 104:123-146

78. Vergani P, Lockless SW, Nairn AC, Gadsby DC (2005) CFTR channel opening by ATP-driven tight dimerization of its nucleotide-binding domains. Nature 433:876-880

79. Vergani P, Nairn AC, Gadsby DC (2003) On the mechanism of MgATP-dependent gating of CFTR $\mathrm{Cl}(-)$ channels. J Gen Physiol 121:17-36

80. Ward CL, Omura S, Kopito RR (1995) Degradation of CFTR by the ubiquitin-proteasome pathway. Cell 83:121-127

81. Wilkinson DJ, Strong TV, Mansoura MK, Wood DL, Smith SS, Collins FS, Dawson DC (1997) CFTR activation: additive effects of stimulatory and inhibitory phosphorylation sites in the $\mathrm{R}$ domain. Am J Physiol 273:L127-L133

82. Zeltwanger S, Wang F, Wang GT, Gillis KD, Hwang TC (1999) Gating of cystic fibrosis transmembrane conductance regulator chloride channels by adenosine triphosphate hydrolysis. Quantitative analysis of a cyclic gating scheme. J Gen Physiol 113:541-554 